\title{
Similarity properties in the dynamics of delayed-feedback semiconductor lasers
}

\author{
Xavier Porte, ${ }^{*}$ Miguel C. Soriano, and Ingo Fischer \\ Instituto de Física Interdisciplinar y Sistemas Complejos, IFISC (CSIC-UIB), Campus Universitat Illes Balears, \\ E-07122 Palma de Mallorca, Spain
}

(Received 9 September 2013; published 18 February 2014)

\begin{abstract}
Semiconductor lasers with delayed feedback exhibit two fundamentally different dynamical states: weak and strong chaos. We characterize experimentally the mechanism for the emergence of strong chaos. Based on these insights, we demonstrate similarity properties for long delays, i.e., similar dynamics for different pump currents when adjusting the feedback strength. For different delay times, even the same time- and amplituderescaled version of the dynamics can be generated. Using a simple rate-equation model, these properties can be corroborated. The results have major consequences for the characterization and tailoring of the dynamics for applications.
\end{abstract}

DOI: 10.1103/PhysRevA.89.023822

\section{INTRODUCTION}

The dynamics of semiconductor lasers subject to delayed feedback has been studied for several decades. Dynamical instabilities in these systems were identified in the 1970's [1] and have been studied since then with various motivations. Initially considered a nuisance, because they have been disturbing many applications from telecommunication to optical storage systems, lasers with delayed feedback were later recognized as excellent testbed systems to study dynamical effects in delay systems [2]. With the growing understanding of the dynamics and its control, the delayed-feedback-induced dynamics has nowadays become more and more attractive for a variety of applications, comprising encrypted communication with chaotic carriers [3], light sources with tunable coherence length [4], ultrafast random bit sequence generation [5], and neuroinspired information processing [6]. Therefore, it is essential to understand the different dynamical properties that can emerge, their underlying mechanisms, and how certain states can be addressed under different conditions. Two fundamentally different chaotic properties, denoted as weak and strong chaos, have recently been distinguished and identified in semiconductor lasers with delayed feedback [7]. In the case of long delays, weak chaos can be synchronized, while strong chaos cannot. They exhibit different scaling properties of the Lyapunov exponents with long delay times, with the Lyapunov exponents characterizing the chaos-specific exponential divergence of nearby trajectories. Either all Lyapunov exponents scale inversely proportional with the delay time, corresponding to weak chaos, or there exists at least one that is independent of the delay time, indicating strong chaos. Recently, it was shown that the normalized autocorrelation function (AC) can be used as an indicator of the weak-strong chaos transition in the laser system with delayed feedback [8].

In this paper, we study the transition from weak to strong chaos by increasing the feedback strength, thereby clarifying the underlying physical mechanism for this transition as the interplay of two characteristic frequencies. These insights have major consequences for a meaningful definition of the weak and moderate feedback regimes. Moreover, in Ref. [8] it was recognized that the position of the maximum of

\footnotetext{
*xavier@ifisc.uib-csic.es
}

PACS number(s): 42.65.Sf, 42.55.Px, 05.45.Jn, 05.45.Pq

the sub-Lyapunov exponent, in dependence on the feedback strength, scales with the pump parameter. Inspired by this finding and by our identified physical mechanism, we study whether the dynamics found in experiments exhibit similarity properties, i.e., whether similar dynamics can be obtained for different parameters. We show that by keeping the ratio of the relaxation oscillation frequency of the solitary laser $f_{\mathrm{RO}}^{S}$ and the maximum feedback-induced frequency shift $\Delta f_{\mathrm{fb}}$ constant, similar AC properties are observed. Keeping also the ratio of $f_{\mathrm{RO}}^{S}$ and the external cavity frequency $f_{\mathrm{EC}}$ constant, the same dynamics, rescaled in amplitude and time, can be generated. Finally, in Sec. III we corroborate these findings using a simple rate-equation-based laser model.

\section{EXPERIMENTAL RESULTS}

For our experiments, we have employed two different quantum well semiconductor lasers: a discrete-mode (DM) laser diode emitting at $1543 \mathrm{~nm}$ [9], and a distributed feedback (DFB) laser emitting at $1541 \mathrm{~nm}$. Without external feedback, the DM laser and the DFB laser have side-mode suppression ratios of more than $40 \mathrm{~dB}$ and longitudinal-mode separations of 150 and $142 \mathrm{GHz}$, respectively. We measured a linewidth enhancement factor of $\alpha \sim 2$ for the DM laser and of $\sim 3$ for the DFB laser using the Henning-Collins approach [10]. The lasers' current and temperature were stabilized to an accuracy of $0.01 \mathrm{~mA}$ and $0.01 \mathrm{~K}$, respectively. Each laser was subject to polarization-maintained optical feedback from a fiber-optic external cavity in the long cavity regime with a round-trip time of $\sim 75 \mathrm{~ns}$. Figure 1 depicts the scheme of the experimental setup, employing standard telecommunication components. We estimated a maximum feedback rate of $\kappa_{\max } \sim 70 \mathrm{~ns}^{-1}$ in our setup. From this maximum value, the feedback rate $\kappa$ could be attenuated by more than $30 \mathrm{~dB}$, with the attenuation defined as Attenuation $[\mathrm{dB}]=-20 \log _{10}\left(\frac{\kappa}{\kappa_{\max }}\right)$. Time series were acquired using a photodiode with $12.5 \mathrm{GHz}$ bandwidth and a $16 \mathrm{GHz}$ analog bandwidth oscilloscope with a sampling rate of $40 \mathrm{GS} / \mathrm{s}$.

To characterize the transition between the different chaotic regimes, we studied the dependence of the $\mathrm{AC}$ on the feedback strength. We analyzed this dependence for the DM laser for four different pump currents: $1.1 I_{\mathrm{th}}, 1.25 I_{\mathrm{th}}$, $1.5 I_{\mathrm{th}}$, and $2 I_{\mathrm{th}}$, with $I_{\text {th }}=12.08 \mathrm{~mA}$ being the solitary laser 


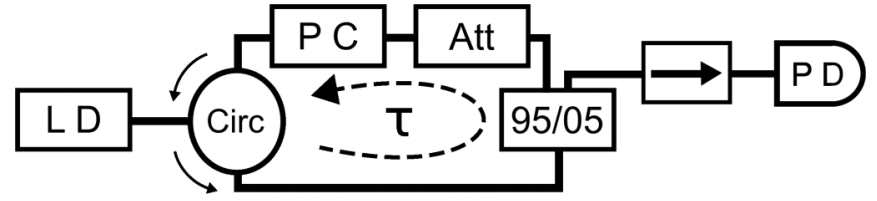

FIG. 1. Scheme of the experimental setup. LD: laser diode; Circ: optical circulator; PC: polarization controller; Att: optical attenuator; 95/05: one by two splitter with $95 \%$ and $5 \%$ coupling ratios; $\rightarrow$ : optical isolator; and PD: photodiode.

threshold. The AC, and especially its behavior around time shifts of $\tau$, is of particular interest, since it illustrates the nonlinear transformation of the delayed-feedback signal by the laser [11]. Moreover, for the laser system, the AC peak height correlates with fundamental chaotic properties related to weak and strong chaos [8]. Figure 2 depicts the maximum of the AC for time shifts of about one delay time $\tau$ for different bias currents and feedback attenuations. In the following we denote the correlations around $\tau$ as the first delay echo. On the abscissa, lower attenuations are depicted on the righthand side (strong feedback) and higher attenuations on the left-hand side (weak feedback). The maximum correlations follow a nonmonotonic dependence on the feedback strength, exhibiting a dip for intermediate feedback strengths. This minimum in the AC curve is an indication of the emergence of strong chaos [8]. The points at the extreme left and right sides, showing correlations lower than 0.2 , correspond to stable emission. The normalized AC then only reflects intensity and detection noise. Nevertheless, the noisy stable emission can be easily distinguished from strong chaos with similarly low correlations via the corresponding time series.

In order to unveil the mechanism that causes this particular dependence we measured the optical spectra with a highresolution optical spectrum analyzer (HROSA) with $10 \mathrm{MHz}$ resolution [12]. The HROSA combines high resolution with a broad scan range. Given that the optical bandwidth of

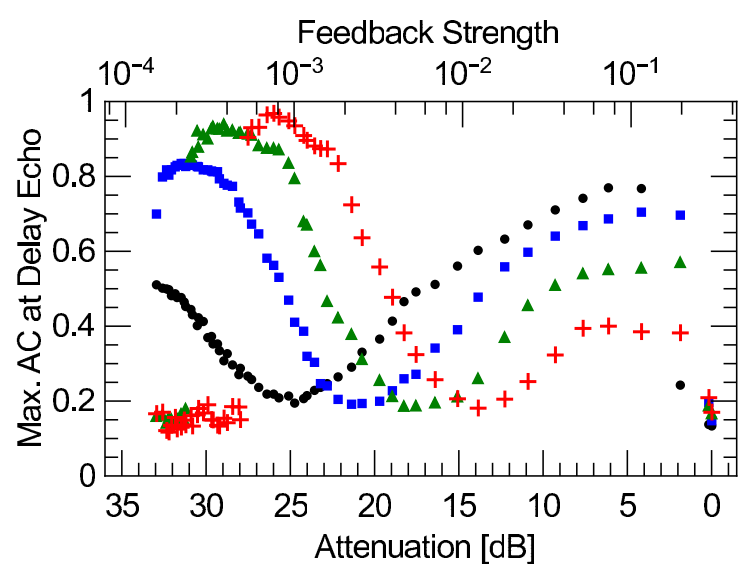

FIG. 2. (Color online) Maximum of the first delay echo as a function of the feedback attenuation for the DM laser for four different operating currents: $1.1 I_{\text {th }}$ (circle), $1.25 I_{\text {th }}$ (square), $1.5 I_{\text {th }}$ (triangle), and $2 I_{\text {th }}$ (cross), respectively. For clarity, we indicate on the top abscissa the feedback strength, i.e., the estimated fraction of emitted power reinjected into the laser.
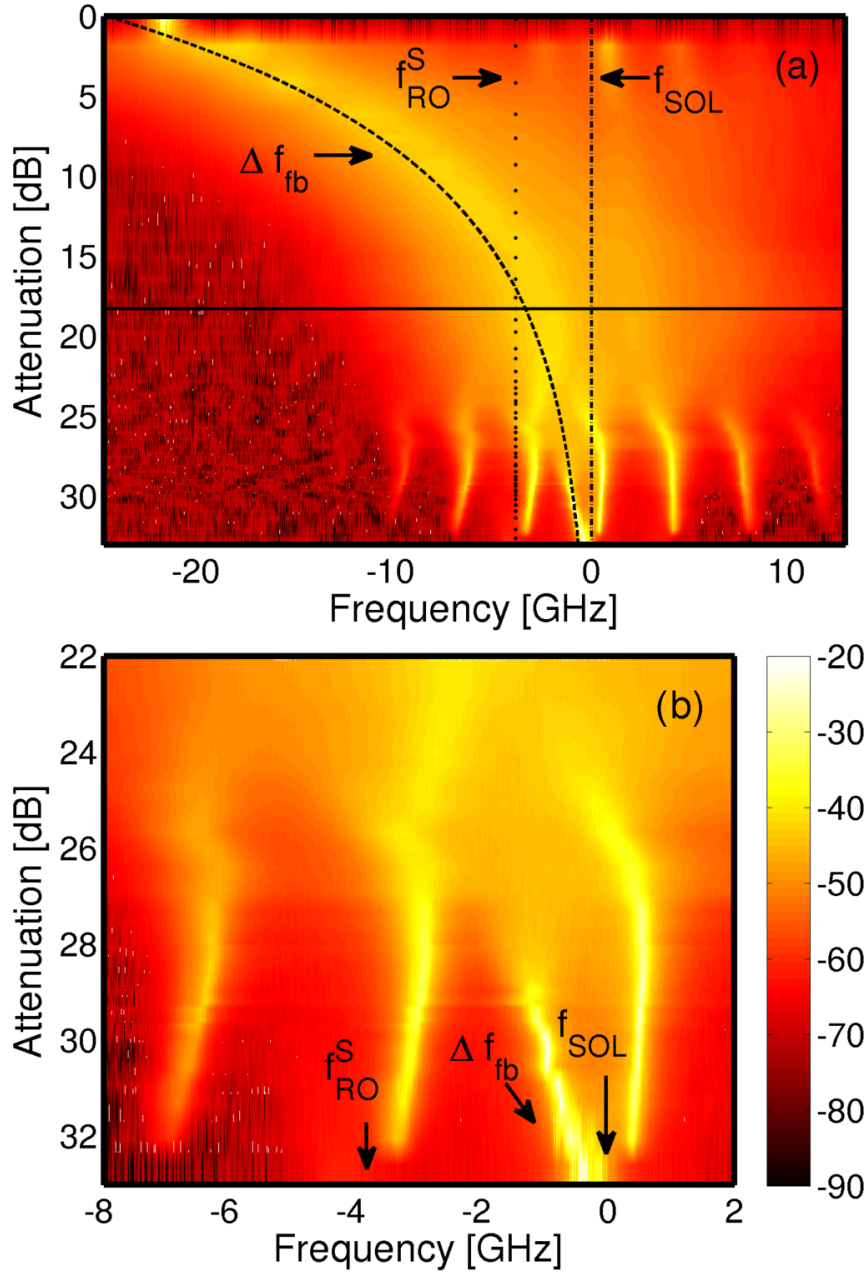

FIG. 3. (Color online) Measured optical spectra vs attenuation for a current of $1.5 I_{\text {th }}$. (a) Vertical dashed-dotted and dotted lines indicate the solitary laser frequency $\left(f_{\mathrm{SOL}}\right)$ and the corresponding relaxation oscillation frequency $\left(f_{\mathrm{RO}}^{S}\right)$, respectively. The dashed curve denotes the position of the spectrally shifted feedback-induced highgain region. The horizontal solid line indicates the attenuation for which we observed the smallest first delay echo. (b) is a zoom of the region close to the solitary frequency for weak feedback conditions.

the laser easily increases by more than three orders of magnitude under the influence of optical feedback [13,14], this combination is essential to detect the interaction between the delayed-feedback dynamics and the relaxation oscillation laser dynamics with good accuracy over a wide range of feedback strengths. Figure 3 depicts the stacked optical spectra for $I=1.5 I_{\text {th }}$ as a function of the feedback attenuation, i.e., each color-coded horizontal line corresponds to an experimentally recorded optical spectrum for a given attenuation value. Figure 3(a) illustrates the transition from low feedback strengths (bottom) to high feedback strengths (top). Below the maximum attenuation the laser exhibits stable emission in its solitary frequency. A slight increase of the feedback strength causes undamping of the relaxation oscillations [15-17], subsequently entering the weak chaos regime. Further increase of the feedback strength causes the transitions towards strong chaos for intermediate feedback. Finally, for the strongest feedback conditions, the laser emission becomes stable again, 
emitting in the high-gain modes region. The vertical dotted line marks the relaxation oscillation frequency without feedback $\left(f_{\mathrm{RO}}^{S}\right)$, which was measured for this pump current to be $\sim 4$ GHz. The dashed curve is a fit that marks the maximum feedback-induced frequency shifts, which have a square-root dependence with the feedback attenuation. The dependence of this shift agrees well with the one derived from a rate-equation model $\Delta f_{\mathrm{fb}}=\alpha \kappa / 2 \pi \quad[18,19]$. The solid horizontal line depicts the attenuation for which the AC exhibits a minimum at $1.5 I_{\text {th }}$, i.e., the region of strong chaos. Remarkably, strong chaos occurs in the region in which $\Delta f_{\mathrm{fb}}$ approaches $f_{\mathrm{RO}}^{S}$, resulting in strong nonlinear interactions.

For a more detailed analysis, Fig. 3(b) shows an enlargement of the region around the solitary laser frequency for weak to intermediate feedback strengths. One can recognize that increasing the feedback strength induces a slight drift of the solitary laser frequency and its corresponding relaxation oscillation. More importantly, for large feedback attenuations $>29 \mathrm{~dB}$, the maximum feedback-induced frequency shift is clearly visible in the range $\{-2$ to 0$\} \mathrm{GHz}$. For smaller feedback attenuations $<29 \mathrm{~dB}$ it starts to nonlinearly mix with the relaxation oscillations, resulting in a qualitative change of the optical spectrum and the associated dynamics. The optical spectrum does not exhibit pronounced relaxation oscillation peaks anymore and is rather broadband. This corresponds to the drop in the AC and can also be linked to the transition to strong chaos along the slope. We suggest to define this transition, when the features of the relaxation oscillations disappear, as the separation of the weak and intermediate feedback regimes.

This interplay between the relaxation oscillations and the delay-induced dynamics suggests that the resulting dynamics might be comparable for different feedback and pump parameters, as long as the ratio of relaxation oscillation frequency $f_{\mathrm{RO}}^{S}$ and maximum feedback-induced frequency shift $\Delta f_{\mathrm{fb}}$ is kept constant. Rescaling the feedback attenuation accordingly defines comparable conditions for the different recorded curves in Fig. 2 . We choose the curve at $2 I_{\text {th }}$ as a reference and rescale the feedback attenuation for the other currents such that the ratios $f_{\mathrm{RO}}^{S} / \Delta f_{\mathrm{fb}}$ correspond. Figure 4(a) shows the resulting curves versus the rescaled feedback attenuation. After rescaling, the U-shaped region of the minimum of the different curves fall on top of each other. The deviations for weak feedback can be mostly attributed to the different signalto-noise ratios (SNR) for different currents. In particular, we have evaluated the influence of the SNR via adding white noise to our time series. The obtained dependence for the AC height on the SNR is in good agreement with the experimental findings, within error margins below 5\%. The deviations at strong feedback can be associated with the onset of alternation between chaotic dynamics and stable emission. This effect is very prominent in the DM laser due to its low $\alpha$ parameter. Altogether, the rescaling and similarity properties of the delayed-feedback-induced dynamics are obtained model independent and are found to hold over large ranges of feedback strengths. In good approximation, the relaxation oscillation frequency exhibits a square-root dependence on the excess pump current over threshold $p_{\text {exc }}=$ $\left(\frac{I}{I_{\mathrm{th}}}-1\right)$. Then, the rescaling corresponds to the condition $f_{\mathrm{RO}}^{S} / \Delta f_{\mathrm{fb}} \sim \sqrt{p_{\mathrm{exc}}} / \kappa$.

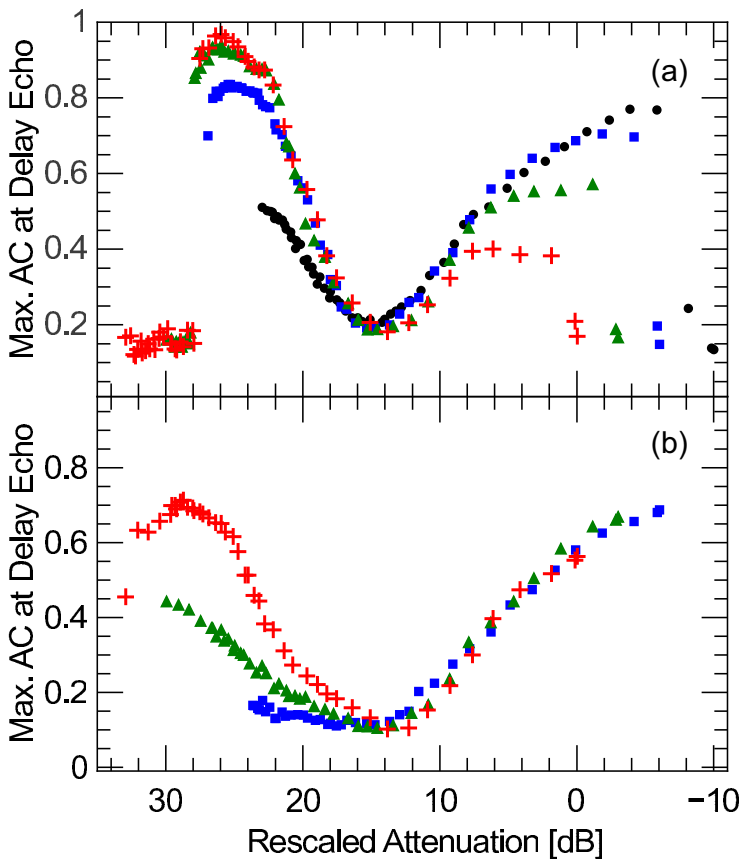

FIG. 4. (Color online) Maximum of the first delay echo as a function of the feedback attenuation for (a) four different operating currents of the DM laser and (b) three different operating currents of the DFB laser. The pump currents are $1.1 I_{\text {th }}$ (circle), $1.25 I_{\text {th }}$ (square), $1.5 I_{\text {th }}$ (triangle), and $2 I_{\text {th }}$ (cross), respectively.

In order to prove the generality of the similarity properties of the delay-induced dynamics, we investigated the rescaling of the feedback strength on the AC for a different laser, keeping the rest of the experimental setup unchanged. Figure 4(b) shows the results for the DFB laser diode under the corresponding change of scale of the feedback attenuation axis. Also for this laser, the different curves fall on top of each other after the rescaling of the feedback attenuation. Again, the deviations for weak feedback can be explained solely by the different SNR for different currents. This device has a higher $\alpha$ parameter than the DM laser and, therefore, stable emission under strong feedback conditions is not observed. Consequently, the curves coincide up to the strongest feedback conditions. The similarity of the dynamical properties of the laser with optical feedback for rescaled feedback strengths indicates that the transitions between different dynamical regimes are not directly linked to an absolute feedback strength as traditionally considered [20,21], but rather to the interplay between feedback strength and relaxation oscillation frequency (pump current). An intriguing consequence of these results is that in order to study the dynamical properties of semiconductor lasers with delayed feedback, one can significantly reduce the amount of measurements in parameter space.

The rescaling considered so far only considers the dependence of the maximum of the first delay echo on pump parameter and feedback strength. Exploring further the similarity properties of this delay system, the delay time plays a crucial role as well. Keeping also the ratio of the relaxation oscillation and the external cavity frequency constant, i.e., $f_{\mathrm{RO}}^{S} / f_{\mathrm{EC}} \sim \tau \sqrt{p_{\mathrm{exc}}}=$ const, we demonstrate that completely equivalent, but amplitude- and time-rescaled, dynamics is obtained. Therefore, we repeat the experiment for a different 


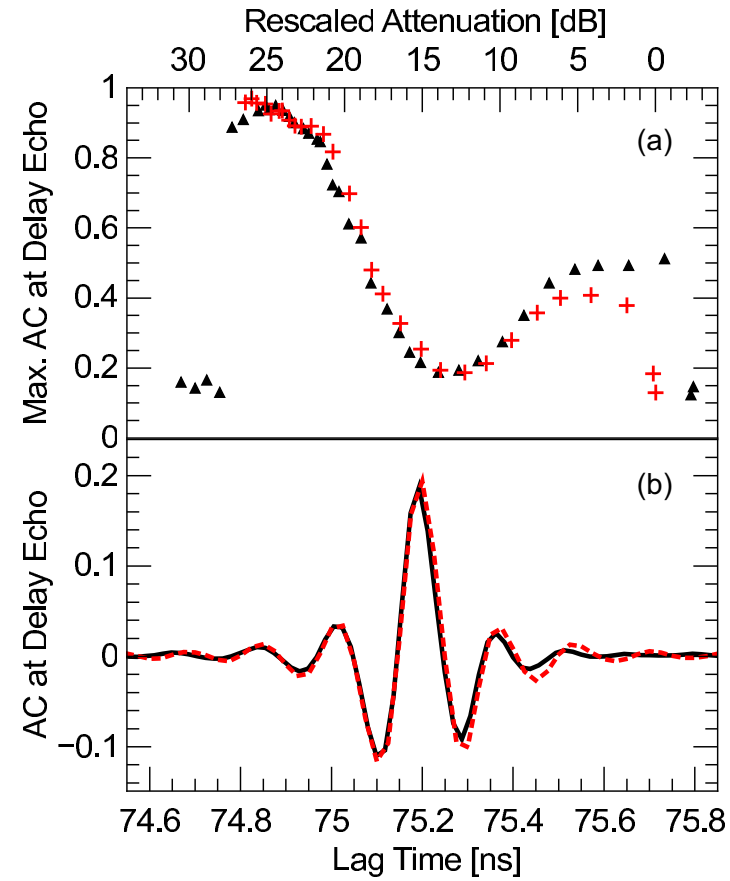

FIG. 5. (Color online) (a) Maximum of the first delay echo and (b) full first delay echo for two different dynamical conditions. Crosses (a) and the dashed line (b) correspond to $2 I_{\mathrm{th}}, \tau_{1}=75.18 \mathrm{~ns}$. Triangles (a) and the solid line (b) correspond to $1.566 \mathrm{I}_{\mathrm{th}}, \tau_{2}=$ $99.95 \mathrm{~ns}$. Both curves in (b) correspond to attenuations at the minimum of each respective AC curve in (a). The time axis is rescaled by $\Delta t^{\prime}=\frac{\tau_{1}}{\tau_{2}} \Delta t$ for the solid line in (b).

delay time $\tau_{2}$. Please note that, in addition to the rescaling in time, the amplitudes also rescale with the corresponding change in the pump current. We extended the length of the external optical cavity by adding $5 \mathrm{~m}$ of fiber, and characterized the dependence of the $\mathrm{AC}$ function on the attenuation for the rescaled current. We change the delay time from $\tau_{1}=$ $75.18 \mathrm{~ns}$ to $\tau_{2}=99.95 \mathrm{~ns}$, corresponding to a scaling factor of $\beta=\frac{\tau_{1}}{\tau_{2}}=0.75$. For these two cases the pump current of $I_{1}=2 I_{\text {th }}$ is compared to a pump current of $I_{2}=1.566 I_{\mathrm{th}}$, respectively, such that $f_{\mathrm{RO}}^{S} / f_{\mathrm{EC}}$ remains equal. The similarity of the dynamics after a change in the delay time is illustrated in Fig. 5. Remarkably, not only is the maximum of the AC around the delay times invariant, as shown in Fig. 5(a), but so is the time-rescaled AC function around the delay times, as depicted in the lower panel [both curves correspond to the minimum at $-14 \mathrm{~dB}$ in Fig. 5(a)] . Moreover, we have verified that all time-rescaled AC functions correspond. From these findings, we can deduce the following similarity property for the autocorrelation function:

$$
\mathrm{AC}\left(\Delta t ; \tau, p_{\mathrm{exc}}, \kappa\right) \cong \mathrm{AC}\left(\beta \Delta t ; \beta \tau, \frac{p_{\mathrm{exc}}}{\beta^{2}}, \frac{\kappa}{\beta}\right) .
$$

This is of high relevance, since this means that a particular dynamics observed for a certain delay time can be simply slowed down or accelerated by appropriately changing the delay time, feedback attenuation, and pump parameter. By this, dynamical properties can be adjusted and tailored for applications such as photonic information processing [6].

\section{NUMERICAL MODELING}

To corroborate our findings, we have employed a simple rate-equation-based laser model to verify the observed similarity properties in the chaotic dynamics regime. A semiconductor laser subject to moderate optical feedback can, in many cases, be described by the Lang-Kobayashi (LK) equations as follows [22-24]:

$$
\begin{gathered}
\dot{E}(t)=\frac{1+i \alpha}{2} G_{\mathrm{N}} n(t) E(t)+\kappa E(t-\tau) e^{-i \omega_{0} \tau}, \\
\dot{n}(t)=p_{\mathrm{exc}} J_{\mathrm{th}}-\gamma n(t)-\left[\Gamma+G_{\mathrm{N}} n(t)\right]|E(t)|^{2},
\end{gathered}
$$

where $E$ and $n$ are the complex electric field amplitude and the carrier number above threshold, respectively. The feedback is accounted for via the delay time $\tau$ and the feedback rate $\kappa$. The parameters in Eq. (1) are the linewidth enhancement factor $(\alpha)$, the differential optical gain $\left(G_{\mathrm{N}}=2.142 \times 10^{4} \mathrm{~s}^{-1}\right)$, and the laser solitary frequency $\left(\omega_{0}\right)$. In Eq. (2), $p_{\text {exc }}$ is the excess pump current over threshold $\left(\frac{J}{J_{\mathrm{th}}}-1\right), J_{\mathrm{th}}=1.552 \times 10^{17} \mathrm{~s}^{-1}$ denotes the pump threshold current in units of the electron charge, $\gamma=0.909 \times 10^{9} \mathrm{~s}^{-1}$ is the carrier decay rate, and $\Gamma=$ $0.357 \times 10^{12} \mathrm{~s}^{-1}$ is the cavity decay rate. Parameter values have been chosen according to Refs. [23,24], except for $\alpha=3$, $\tau=10 \mathrm{~ns}$. The feedback rate $\kappa$ has been varied in the range $1-$ $100 \mathrm{~ns}^{-1}$. In this model, the solitary laser relaxation oscillation frequency is given by $f_{\mathrm{RO}}=\frac{1}{2 \pi} \sqrt{G_{\mathrm{N}} p_{\mathrm{exc}} J_{\mathrm{th}}}$ and the maximum feedback-induced frequency shift is $\Delta f_{\mathrm{fb}}=\alpha \kappa / 2 \pi$.

Figure 6 depicts the numerical results for the height of the first AC peak for different pump currents. We find that the curves for the AC peak height become pump current
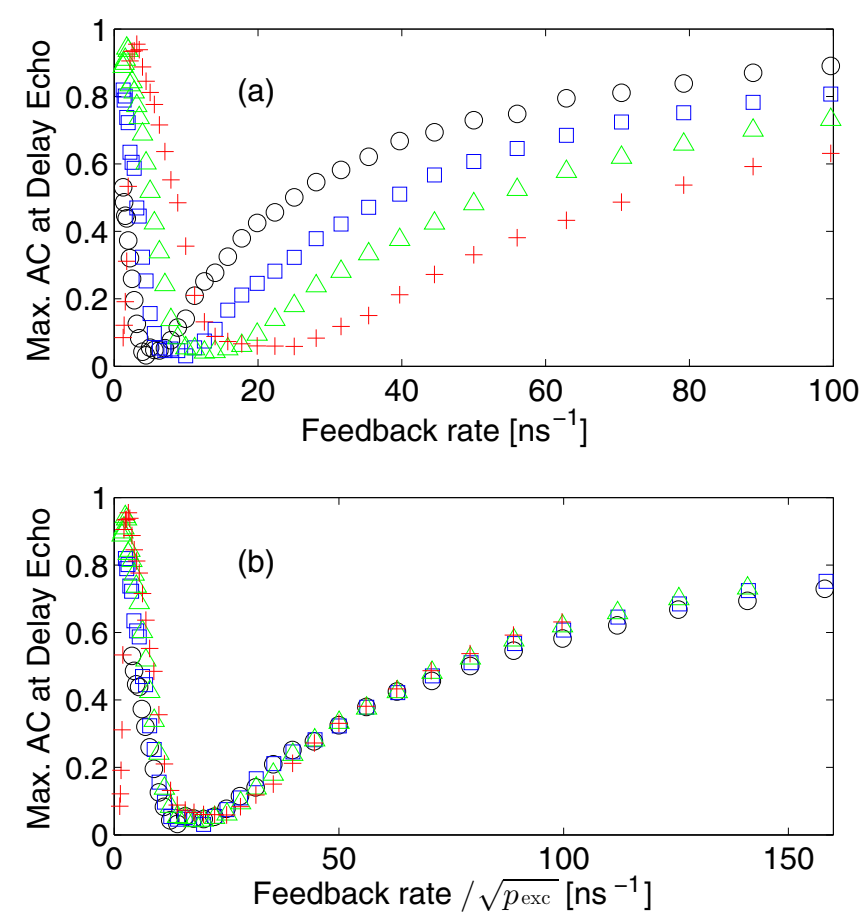

FIG. 6. (Color online) Maximum of the first autocorrelation peak as a function of the feedback attenuation for four different operating currents: $1.1 I_{\text {th }}$ (circle), $1.25 I_{\text {th }}$ (square), $1.5 I_{\text {th }}$ (triangle), and $2 I_{\text {th }}$ (cross), respectively. In (b) the feedback rate has been scaled as $\sqrt{p_{\text {exc }}}$. 

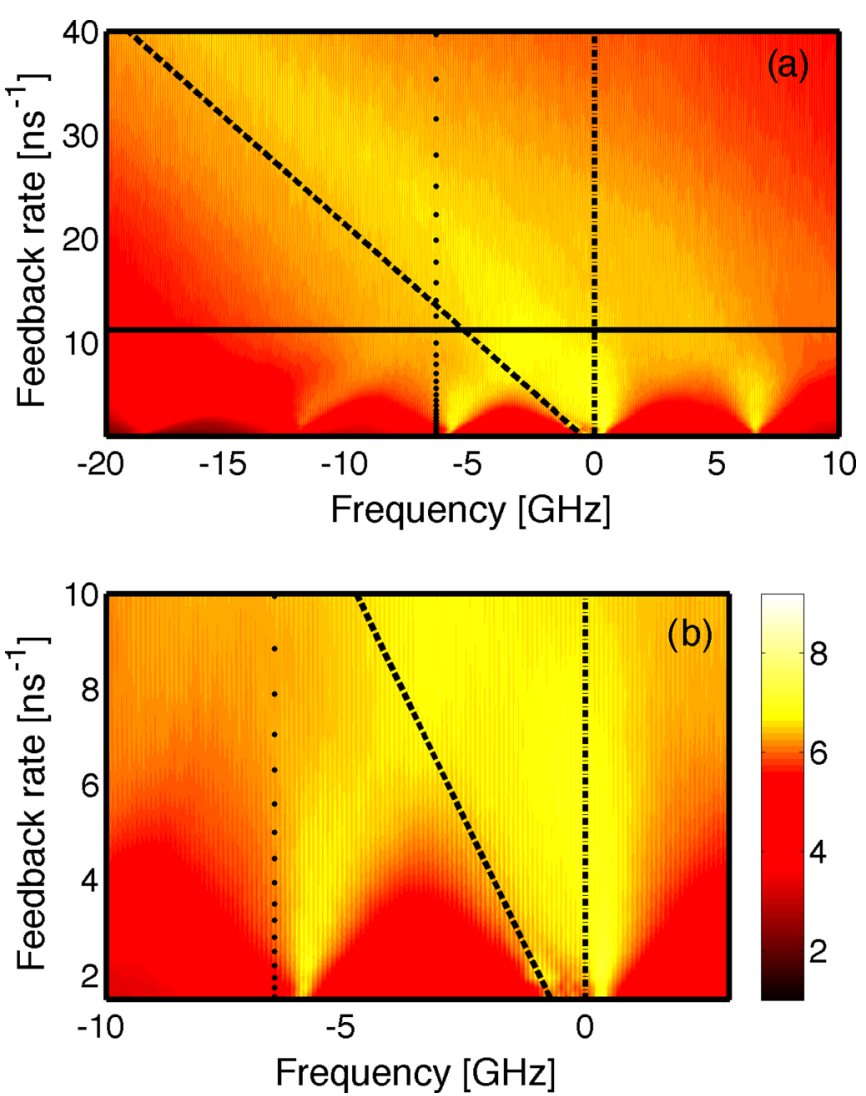

FIG. 7. (Color online) Color-coded amplitude of the numerically calculated optical spectra vs feedback rate for a pump of $p_{\text {exc }}=0.5$ $\left(I=1.5 I_{\text {th }}\right)$. The vertical dashed-dotted and dotted lines indicate the solitary laser frequency and its relaxation oscillation frequency, respectively. The horizontal solid line indicates the feedback for which we have the minimum of the $\mathrm{AC}$, and the oblique dashed line depicts the position of $\Delta f_{\mathrm{fb}}$. (b) is a zoom of the region close to the solitary laser frequency for low feedback strengths of (a).

independent when the feedback rate $\kappa$ is rescaled by $\sqrt{p_{\text {exc }}}$. This agrees with the scaling of the Lyapunov exponents as reported in Ref. [8] in the approximation of weak feedback and small variations of $n$.

In order to explore the generality of our experimental results in more detail, we have numerically computed the optical spectra of the laser with feedback for different parameters as a function of the feedback rate $\kappa$. Figure 7(a) depicts the stacked optical frequency spectra for $p_{\text {exc }}=0.5$ when the feedback rate is varied in Eq. (1). For increasing feedback, the laser exhibits a transition from stable emission to undamped relaxation oscillations such as in the experiments. Further increasing the feedback, the frequency bands corresponding to the solitary laser emission (see the dashed-dotted line in Fig. 7), the relaxation oscillations (dotted line), and its higher harmonics become wider, starting to nonlinearly mix, until they can no longer be distinguished. The minimum in the height of the AC peak [the horizontal line in Fig. 7(a)] occurs just after the onset of the strong mixing of the frequency bands. In particular, the minimum $\mathrm{AC}$ echo occurs when $\Delta f_{\mathrm{fb}}$ (oblique line in Fig. 7) approaches the relaxation oscillation frequency of the solitary laser. We systematically find the same qualitative behavior of the dynamics for all currents.

\section{CONCLUSIONS}

We have shown in experiments and simulations that the dynamics of a laser with delayed feedback shows similarity properties. For different feedback strengths, pump parameters, and delay times, equivalent dynamics was observed. This is corroborated by the fact that, by adjusting delay time and feedback strength according to the change in pump current, the ratio between the total number of external cavity modes and maximum feedback-induced frequency shift remains constant. Our studies also indicate that the compensation of some parameters by others has its limitations. Different $\alpha$ parameters cannot be compensated for and will always result in different dynamics. However, we note that even for the same laser, different $\alpha$ parameters can be realized [25]. This study, together with the identification of the physical mechanism that generates strong chaos in semiconductor lasers, opens a clarifying perspective onto the broad field of delay-coupled semiconductor lasers. Our results offer promising implications for future photonics applications, in which nonlinear delayedfeedback dynamics is used.

\section{ACKNOWLEDGMENTS}

The authors acknowledge fruitful discussions with T. Jüngling, O. D’Huys, D. Brunner, R. Toral, E. HernándezGarcía, and C. R. Mirasso. This work was supported by MINECO (Spain), Comunitat Autònoma de les Illes Balears, FEDER, and the European Commission under Projects TEC2012-36335 (TRIPHOP), Grups Competitius, and EC FP7 Projects PHOCUS (Grant No. 240763).
[1] C. Risch and C. Voumard, J. Appl. Phys. 48, 2083 (1977).

[2] M. C. Soriano, J. García-Ojalvo, C. R. Mirasso, and I. Fischer, Rev. Mod. Phys. 85, 421 (2013).

[3] A. Argyris, D. Syvridis, L. Larger, V. Annovazzi-Lodi, P. Colet, I. Fischer, J. Garcia-Ojalvo, C. R. Mirasso, L. Pesquera, and K. A. Shore, Nature (London) 438, 343 (2005).

[4] M. Peil, I. Fischer, W. Elsäßer, S. Bakic, N. Damaschke, C. Tropea, S. Stry, and J. Sacher, Appl. Phys. Lett. 89, 091106 (2006).
[5] A. Uchida, K. Amano, M. Inoue, K. Hirano, S. Naito, H. Someya, I. Oowada, T. Kurashige, M. Shiki, S. Yoshimori, K. Yoshimura, and P. Davis, Nat. Photonics 2, 728 (2008).

[6] D. Brunner, M. C. Soriano, C. R. Mirasso, and I. Fischer, Nat. Commun. 4, 1364 (2013).

[7] S. Heiligenthal, T. Dahms, S. Yanchuk, T. Jüngling, V. Flunkert, I. Kanter, E. Schöll, and W. Kinzel, Phys. Rev. Lett. 107, 234102 (2011). 
[8] S. Heiligenthal, T. Jüngling, O. D’Huys, D. A. Arroyo-Almanza, M. C. Soriano, I. Fischer, I. Kanter, and W. Kinzel, Phys. Rev. E 88, 012902 (2013).

[9] B. Kelly, R. Phelan, D. Jones, C. Herbert, J. O'Carroll, M. Rensing, J. Wendelboe, C. B. Watts, A. KaszubowskaAnandarajah, P. Perry, C. Guignard, L. P. Barry, and J. O'Gorman, Electron. Lett. 43, 1282 (2007).

[10] I. D. Henning and J. V. Collins, Electron. Lett. 19, 927 (1983).

[11] G. Van der Sande, M. C. Soriano, I. Fischer, and C. R. Mirasso, Phys. Rev. E 77, 055202(R) (2008).

[12] A. Villafranca, J. A. Lázaro, I. Salinas, and I. Garcés, IEEE Photon. Technol. Lett. 17, 2268 (2005).

[13] D. Lenstra, B. Verbeek, and A. Den Boef, IEEE J. Quantum Electron. 21, 674 (1985).

[14] D. Brunner, X. Porte, M. C. Soriano, and I. Fischer, Sci. Rep. 2, 732 (2012)

[15] A. Hohl and A. Gavrielides, Phys. Rev. Lett. 82, 1148 (1999).
[16] J. Mørk, J. Mark, and B. Tromborg, Phys. Rev. Lett. 65, 1999 (1990).

[17] J. Ye, H. Li, and J. G. McInerney, Phys. Rev. A 47, 2249 (1993).

[18] C. H. Henry and R. F. Kazarinov, IEEE J. Quantum Electron. 22, 294 (1986).

[19] A. M. Levine, G. H. M. van Tartwijk, D. Lenstra, and T. Erneux, Phys. Rev. A 52, R3436 (1995).

[20] R. W. Tkach and A. R. Chraplyvy, IEEE J. Lightwave Technol. 4, 1655 (1986).

[21] K. Petermann, IEEE J. Sel. Top. Quantum Electron. 1, 480 (1995).

[22] R. Lang and K. Kobayashi, IEEE J. Quantum Electron. 16, 347 (1980).

[23] G. H. M. van Tartwijk, A. M. Levine, and D. Lenstra, IEEE J. Sel. Top. Quantum Electron. 1, 466 (1995).

[24] V. Ahlers and U. Parlitz, and W. Lauterborn, Phys. Rev. E 58, 7208 (1998).

[25] T. Heil, I. Fischer, and W. Elsäßer, Phys. Rev. A 60, 634 (1999). 